\title{
On invariants of polynomial functions, II Y. Fukuma
}

\author{
Communicated by V. A. Artamonov
}

\begin{abstract}
A BStRACT. Let $P$ be a finite partially ordered set. In our previous paper, we defined the sectional geometric genus $g_{i}(P)$ of $P$ and studied $g_{i}(P)$. In this paper, by using this sectional geometric genus of $P$, we will give a criterion about the case in which $P$ has no order.
\end{abstract}

\section{Introduction}

In our previous paper [1], we studied polynomial functions. In particular, we generalized the notion of the $i$ th sectional geometric genus of polarized varieties, which is an important invariant of polarized varieties (see [2, Definition 3.2]), to the case of polynomial functions. Here we note that if $i=1$, then the first sectional geometric genus of any polynomial function in two variables associated with a polynomial function $h$ is equal to the sectional genus of $h$ which was defined by Ooishi [5, Definition 1.3] (see Remark 2). By using this invariant, we proved the following theorem about partially ordered sets (see [1, Theorem 4.1]).

Theorem 1. Let $P$ be a finite partially ordered set, and let $i$ be an integer with $1 \leqslant i \leqslant d(P)$. Then $g_{i}(P)=0$ if and only if $l(P) \geqslant d(P)-i$, where $d(P)=\sharp(P)$ and $l(P)$ denotes the length of $P$ (see Definition 6 (3)).

As a corollary, we can get the following result which gives a neccesary and sufficient condition for being totally ordered.

This research was supported by JSPS KAKENHI (grant no. 16K05103).

2020 MSC: 05A10, 05A15, 06A06.

Key words and phrases: partially ordered set, order polynomial, polynomial function, sectional geometric genus. 
Corollary 1. Let $P$ be a finite partially ordered set. Then $g_{1}(P)=0$ if and only if $P$ is a totally ordered set.

In this paper, by using the sectional geometric genus of a finite partially ordered set $P$, we will give two criterions about the case in which $P$ has no order (Theorems 5 and 6).

\section{Preliminaries}

Notation 1. For a real number $m$ and a non-negative integer $n$, let

$$
[m]_{n}= \begin{cases}m(m-1) \cdots(m-n+1) & \text { if } n \geqslant 1, \\ 1 & \text { if } n=0 .\end{cases}
$$

Then for $n$ fixed, $[t]_{n}$ is a polynomial in $t$ whose degree is $n$. For any non-negative integer $n$,

$$
n !:= \begin{cases}{[n]_{n}} & \text { if } n \geqslant 1, \\ 1 & \text { if } n=0 .\end{cases}
$$

Assume that $m$ and $n$ are non-negative integers. Then we put

$$
\left(\begin{array}{c}
m \\
n
\end{array}\right):= \begin{cases}\frac{[m]_{n}}{n !} & \text { if } m \geqslant n \\
0 & \text { if } m<n .\end{cases}
$$

We note that

$$
\left(\begin{array}{c}
m \\
0
\end{array}\right)=1 \text { if } m \geqslant 0 .
$$

Definition 1 (see $[4, \S 1]$ ). (1) Let $f: \mathbb{Z} \rightarrow \mathbb{Z}$ be a function. Then $f$ is called a polynomial function if $f$ satisfies the following.

(A) There exist an integer $N_{1}$ and a polynomial $P(n) \in \mathbb{C}[n]$ such that $f(n)=P(n)$ for every integer $n$ with $n>N_{1}$.

(B) There exists an integer $N_{2}$ such that $f(m)=0$ for every integer $m$ with $m<N_{2}$.

In this case we put $P_{f}(t):=P(t)$ because $P(t)$ depends on the function $f$. We call this polynomial $P_{f}(t)$ the polynomial associated with $f$.

(2) Let $\phi(t) \in \mathbb{C}\left[t, t^{-1}\right]$ and we put $\phi(t)=\sum_{i} a_{i} t^{i}$. Then we put

$$
d(\phi):=\max \left\{k \mid a_{k} \neq 0\right\} .
$$

(3) Let $f$ be a polynomial function such that $P_{f}(t) \neq 0_{\mathbb{C}[t]}$. Then we put $d(f):=d\left(P_{f}\right)$. 
Remark 1. Let $f$ be a polynomial function. Then $P_{f}(t) \in \mathbb{Q}[t]$ (see [1, Remark $2.1(1)]$ ).

Notation 2. (1) Let $f: \mathbb{Z} \rightarrow \mathbb{Z}$ be a function. We put

$$
\nabla f(n):=\sum_{i \leqslant n} f(i) \quad \text { and } \quad F_{f}(t):=\sum_{n \in \mathbb{Z}} f(n) t^{n} .
$$

(2) $\mathcal{P F}$ denotes the set of polynomial functions, and

$$
\mathcal{P} \mathcal{F}^{\geqslant 0}:=\{g(t) \in \mathcal{P} \mathcal{F} \mid g(t)=0 \text { for } \forall t<0\} .
$$

Notation 3. Let $f: \mathbb{Z} \times \mathbb{Z} \rightarrow \mathbb{Z}$ be a function in two variables. We put $f_{1}(x):=f(x, 0)$ and $f_{2}(y):=f(0, y)$.

Definition 2. Let $h(x)$ be a polynomial function in $x$. Then a function $f: \mathbb{Z} \times \mathbb{Z} \rightarrow \mathbb{Z}$ is called a polynomial function in two variables associated with $h(x)$ if the following hold.

(1) $f_{1}(x)=h(x)$.

(2) There exists an integer $N$ such that $f_{2}(y)=0$ for every integer $y$ with $y \leqslant N$.

The following lemma is well-known:

Lemma 1. Let $p(t) \in \mathbb{C}[t]$ be a polynomial in $t$ such that $p(t) \neq 0_{\mathbb{C}[t]}$ and $p(n) \in \mathbb{Z}$ for any integer $n$, and let $k$ be the degree of $p(t)$. Then for every integer $d$ with $0 \leqslant k \leqslant d$ there exists a unique sequence of integers $\left(b_{0}, b_{1}, \ldots, b_{d}\right)$ such that the following holds.

$$
p(t)=\sum_{j=0}^{d}(-1)^{j} b_{j}\left(\begin{array}{c}
t+d-j \\
d-j
\end{array}\right) .
$$

Notation 4. Let $h(t) \in \mathbb{C}[t]$ be a polynomial in $t$ such that $h(t) \neq 0_{\mathbb{C}[t]}$ and $h(n) \in \mathbb{Z}$ for every integer $n$. We put $k=\operatorname{deg} h(t)$, and let $d$ be an integer with $k \leqslant d$. Then by Lemma 1 , there exists a unique sequence of integers $\left(b_{0}, b_{1}, \ldots, b_{d}\right)$ such that the following holds.

$$
h(t)=b_{0}\left(\begin{array}{c}
t+d \\
d
\end{array}\right)-b_{1}\left(\begin{array}{c}
t+d-1 \\
d-1
\end{array}\right)+\cdots+(-1)^{d} b_{d} .
$$

Here we put $e_{i}^{k, d}(h(t)):=b_{i}$, and if $k=d$, then we put $e_{i}(h(t)):=e_{i}^{d, d}(h(t))$. Here we note that if $k<d$, then $e_{i}^{k, d}(h(t))=0$ for every integer $i$ with $0 \leqslant i \leqslant d-k-1$. 
Definition 3. (1) Let $h(t) \in \mathbb{C}[t]$ be a polynomial in $t$ such that $h(t) \neq$ $0_{\mathbb{C}[t]}$ and $h(n) \in \mathbb{Z}$ for every integer $n$. We use Notation 4 . Then for every integer $i$ with $0 \leqslant i \leqslant d(h)$, we define the $i$ th sectional $H$-arithmetic genus $\chi_{i}^{H}(h)$ of $h(t)$ as follows.

$$
\chi_{i}^{H}(h):=\sum_{j=0}^{i}(-1)^{j} e_{j}(h(t)) .
$$

(2) Let $f: \mathbb{Z} \rightarrow \mathbb{Z}$ be a polynomial function, and let $P_{f}(t) \in \mathbb{Q}[t]$ be the polynomial associated with $f$ such that $P_{f}(t) \neq 0_{\mathbb{Q}[t]}$. Then, for every integer $i$ with $0 \leqslant i \leqslant d(f)$, we define the $i$ th sectional $H$-arithmetic genus $\chi_{i}^{H}(f)$ of $f$ as follows.

$$
\chi_{i}^{H}(f):=\chi_{i}^{H}\left(P_{f}\right) .
$$

Definition 4. Let $h(x)$ be a polynomial function in $x$ such that $h(x) \neq$ $0_{\mathbb{Q}[x]}$, and let $f: \mathbb{Z} \times \mathbb{Z} \rightarrow \mathbb{Z}$ be a two variable polynomial function associated with $h(x)$. We use Notations 2 and 3. For every integer $i$ with $0 \leqslant i \leqslant d(h)$, the $i$ th sectional geometric genus $g_{i}(f)$ of $f$ is defined by the following:

$$
g_{i}(f):=(-1)^{i}\left(\chi_{i}^{H}(h)-\nabla f_{2}(0)+\nabla f_{2}(-i)\right) .
$$

Remark 2. Let $f: \mathbb{Z} \times \mathbb{Z} \rightarrow \mathbb{Z}$ be a polynomial function in two variables associated with a polynomial function $h(x)$. If $i=1$ and $d(h) \geqslant 1$, then $g_{1}(f)=g_{s}(h)$, that is, $g_{1}(f)$ is the sectional genus of $h$ (see [1, Remark $3.4(1)])$.

Definition 5. From now on, a partially ordered set is called a poset for short in this paper.

(1) Let $P$ be a finite poset. We put

$$
d(P):=\sharp(P)
$$

and for every $n \in \mathbb{N}$

$$
\Omega(P, n):=\sharp\left\{\sigma: P \rightarrow\{1, \ldots, n\} \mid \sigma\left(x_{i}\right) \leqslant \sigma\left(x_{j}\right) \text { if } x_{i} \leqslant x_{j} .\right\} .
$$

Then $\Omega(P, n)$ is a polynomial in $n$ whose degree is $d(P)$ (see [6, 3.12]). This is called the order polynomial of $P$.

(2) Let $P$ be a finite poset and let $\Omega(P, n)$ be the order polynomial of $P$. We put

$$
h_{P}(x):= \begin{cases}\Omega(P, x) & \text { if } x \in \mathbb{Z} \text { with } 1 \leqslant x \\ 0 & \text { otherwise }\end{cases}
$$


Then $h_{P}$ is a polynomial function, and we define a polynomial function in two variables $f_{P}(x, y)$ associated with $h_{P}(x)$ as follows.

$$
f_{P}(x, y):=h_{P}(x) \cdot \rho(y)
$$

where

$$
\rho(y):= \begin{cases}1 & \text { if } y=0 \\ 0 & \text { otherwise. }\end{cases}
$$

We call $f_{P}(x, y)$ the generalized polynomial function associated with $P$. We note that $d(P)=d\left(h_{P}\right)$.

(3) For every integer $i$ with $0 \leqslant i \leqslant d(P)$ we put $\chi_{i}^{H}(P):=\chi_{i}^{H}\left(h_{P}\right)$ and $g_{i}(P):=g_{i}\left(f_{P}\right)$, which are called the $i$ th sectional $H$-arithmetic genus and the $i$ th sectional geometric genus of $P$ respectively. We note that $g_{1}(P)=g_{s}\left(h_{P}\right)($ see Remark 2$)$.

Definition 6. (1) Let $P$ be a finite poset, and let $C$ be a subset of $P$. Then $C$ is called a chain of $P$ if any two elements of $C$ are comparable.

(2) Let $P$ be a finite poset, and let $C$ be a chain of $P$. Then we put $l(C):=\sharp(C)-1$.

(3) Let $P$ be a finite poset. Then we put

$$
l(P):=\max \{l(C) \mid C \text { is a chain of } P\}
$$

which is called the length of $P$.

(4) Let $n$ be a natural number and let $\Sigma_{n}$ be the set of all permutations of $\{1, \ldots, n\}$. For $\sigma \in \Sigma_{n}$ with

$$
\sigma=\left(\begin{array}{cccc}
1 & 2 & \cdots & n \\
a_{1} & a_{2} & \cdots & a_{n}
\end{array}\right)
$$

we put

$$
\llbracket a_{1}, \ldots, a_{n} \rrbracket:=\left(\begin{array}{cccc}
1 & 2 & \cdots & n \\
a_{1} & a_{2} & \cdots & a_{n}
\end{array}\right) .
$$

(5) Let $P$ be a finite poset. We put $P=\left\{x_{1}, \ldots, x_{d(P)}\right\}$ and

$$
\begin{aligned}
A(P):= & \{\mu: P \rightarrow\{1, \ldots, d(P)\} \mid \\
& \left.\mu \text { is a bijection such that } \mu\left(x_{i}\right)<\mu\left(x_{j}\right) \text { if } x_{i}<x_{j}\right\} .
\end{aligned}
$$

(5.1) We fix an element $\mu \in A(P)$. Then we put

$$
\mathcal{L}(P ; \mu):=\left\{\llbracket \mu \circ \sigma^{-1}(1), \ldots, \mu \circ \sigma^{-1}(d(P)) \rrbracket \mid \sigma \in A(P)\right\} .
$$


By definition, we get that $\mathcal{L}(P ; \mu) \subset \Sigma_{d(P)}$. We call the set $\mathcal{L}(P ; \mu)$ the Jordan-Hölder set of $P$ with respect to $\mu$ (see [6, 3.13]).

(5.2) For $\pi \in \Sigma_{n}$ with $\pi=\left(a_{1}, \ldots, a_{n}\right)$, we put

$$
D(\pi):=\left\{a_{i} \mid a_{i}>a_{i+1}\right\} \quad \text { and } \delta(\pi):=\sharp D(\pi) .
$$

For a natural number $n$ and a subset $S \subset\{1, \ldots, n-1\}$, we put

$$
D_{n}(S):=\left\{\pi \in \Sigma_{n} \mid S=D(\pi)\right\} .
$$

Let $\mu, \mu^{\prime} \in A(P)$ with $\mu \neq \mu^{\prime}$. Then by [6, 3.13.1 Theorem], we obtain the following. For any subset $S \subset\{1, \ldots, d(P)-1\}, \sharp\left(D_{d(P)}(S) \cap\right.$ $\mathcal{L}(P ; \mu))=\sharp\left(D_{d(P)}(S) \cap \mathcal{L}\left(P ; \mu^{\prime}\right)\right)$. In particular, we get that for every integer $i$ with $1 \leqslant i \leqslant d(P)-1$

$$
\sharp\{\pi \in \mathcal{L}(P ; \mu) \mid \delta(\pi)=i\}=\sharp\left\{\pi \in \mathcal{L}\left(P ; \mu^{\prime}\right) \mid \delta(\pi)=i\right\} .
$$

So when we use results concerned with $\delta(\pi)$ (for example, Proposition 1 below), we describe the Jordan-Hölder set as $\mathcal{L}(P)$ instead of $\mathcal{L}(P ; \mu)$ for $\mu \in A(P)$.

Proposition 1. Let $P$ be a finite poset and let $h_{P}(x)$ be the following:

$$
h_{P}(x):= \begin{cases}\Omega(P, x) & \text { if } x \geqslant 1, \\ 0 & \text { otherwise. }\end{cases}
$$

Then

$$
F_{h_{P}}(t)=\left(\sum_{\pi \in \mathcal{L}(P)} t^{1+\delta(\pi)}\right)(1-t)^{-d(P)-1} .
$$

Proof. See [6, 3.15.8 Theorem].

Notation 5. Let $P$ be a finite poset, and let $f_{P}(x, y)$ be the generalized polynomial function associated with $P$. By [1, Theorem 2.1 and Remark $2.4]$ there exists a polynomial $\phi(t) \in \mathbb{Z}[t]$ such that

$$
F_{h_{P}}(t)=\frac{\phi(t)}{(1-t)^{d}},
$$

where $d=d\left(h_{P}\right)+1$. Here we put $F_{f_{P}}(t):=F_{h_{P}}(t), \phi_{f_{P}}(t):=\phi(t)$, and

$$
\phi_{f_{P}}(t)=\sum_{j=0}^{d\left(\phi_{f_{P}}\right)} a_{j} t^{j}
$$

where $a_{j} \in \mathbb{Z}$ for every $j$. Let $a_{j}(P):=a_{j}$. 
Remark 3. We note that $h_{P}(t) \in \mathcal{P} \mathcal{F} \geqslant 0, P_{h_{P}}(t) \neq 0_{\mathbb{Q}[t]}$, and $f_{P}(0, m)=$ $f_{P}(1, m)=0$ for every integer $m$ with $m \neq 0$. Hence by [1, Theorem 3.2] we get the following:

(1) For every integer $i$ with $1 \leqslant i \leqslant d(P)$

$$
g_{i}(P)= \begin{cases}\sum_{k=i+1}^{d\left(\phi_{f_{P}}\right)}\left(\begin{array}{c}
k-1 \\
i
\end{array}\right) a_{k}(P) & \text { if } i+1 \leqslant d\left(\phi_{f_{P}}\right), \\
0 & \text { if } d\left(\phi_{f_{P}}\right) \leqslant i\end{cases}
$$

(2) Since $f_{P}(x, y)$ satisfies the assumptions in [1, Corollary 3.2], we get $g_{i}(P) \geqslant 0$ for every integer $i$ with $1 \leqslant i \leqslant d(P)$. Moreover if $i=d(P)$, then $g_{d(P)}(P)=0$ by Proposition 1 and $(1)$ above.

\section{Main results}

In this section, by using the sectional geometric genus of a poset $P$, we are going to give two criterions about the case in which $P$ has no order.

Theorem 2. Let $d$ and $k$ be nonnegative integers and $S(a, b)$ the Stirling number of the second kind, where $a$ and $b$ are nonnegative integers. Then for every integer $j$ with $0 \leqslant j \leqslant d$ we have

$$
e_{j}^{k, d}\left(t^{k}\right)=(-1)^{d-k}(d-j) ! S(k+1, d+1-j) .
$$

Proof. By Lemma 1 and Notation 4 we have

$$
t^{k}=\sum_{j=0}^{d}(-1)^{j} e_{j}^{k, d}\left(t^{k}\right)\left(\begin{array}{c}
t+d-j \\
d-j
\end{array}\right)
$$

Using this equation (1), we have

$$
\begin{aligned}
(-t)^{k} & =\sum_{j=0}^{d}(-1)^{j} e_{j}^{k, d}\left(t^{k}\right)\left(\begin{array}{c}
-t+d-j \\
d-j
\end{array}\right) \\
& =\sum_{j=0}^{d}(-1)^{j} e_{j}^{k, d}\left(t^{k}\right) \frac{(-t+d-j)(-t+d-j-1) \cdots(-t+1)}{(d-j) !} \\
& =\sum_{j=0}^{d}(-1)^{j}(-1)^{d-j} e_{j}^{k, d}\left(t^{k}\right) \frac{(t-d+j)(t-d+j+1) \cdots(t-1)}{(d-j) !} \\
& =\sum_{j=0}^{d}(-1)^{d} e_{j}^{k, d}\left(t^{k}\right)\left(\begin{array}{c}
t-1 \\
d-j
\end{array}\right) .
\end{aligned}
$$


Hence we have

$$
(-1)^{d-k} t^{k}=\sum_{j=0}^{d} e_{j}^{k, d}\left(t^{k}\right)\left(\begin{array}{l}
t-1 \\
d-j
\end{array}\right) .
$$

Moreover by (2) we have

$$
\begin{aligned}
(-1)^{d-k} t^{k+1} & =\sum_{j=0}^{d} e_{j}^{k, d}\left(t^{k}\right) t\left(\begin{array}{c}
t-1 \\
d-j
\end{array}\right) \\
& =\sum_{j=0}^{d} e_{j}^{k, d}\left(t^{k}\right) \frac{t(t-1) \cdots(t-d+j)}{(d-j) !} \\
& =\sum_{j=0}^{d} e_{j}^{k, d}\left(t^{k}\right) \frac{t(t-1) \cdots(t-d+j)}{(d-j+1) !}(d-j+1) \\
& =\sum_{j=0}^{d}(d-j+1) e_{j}^{k, d}\left(t^{k}\right)\left(\begin{array}{c}
t \\
d-j+1
\end{array}\right) \\
& =\sum_{j=d-k}^{d}(d-j+1) e_{j}^{k, d}\left(t^{k}\right)\left(\begin{array}{c}
t \\
d-j+1
\end{array}\right) \\
& =\sum_{j=0}^{k}(k-j+1) e_{d-k+j}^{k, d}\left(t^{k}\right)\left(\begin{array}{c}
t \\
k-j+1
\end{array}\right) .
\end{aligned}
$$

On the other hand, by $[6,(1.94 \mathrm{~d})]$ we get

$$
\begin{aligned}
t^{k+1} & =\sum_{j=0}^{k+1}(k-j+1) ! S(k+1, k+1-j)\left(\begin{array}{c}
t \\
k-j+1
\end{array}\right) \\
& =\sum_{j=0}^{k}(k-j+1) ! S(k+1, k+1-j)\left(\begin{array}{c}
t \\
k-j+1
\end{array}\right)
\end{aligned}
$$

because $S(k+1,0)=0$.

Therefore we get $e_{d-k+j}^{k, d}\left(t^{k}\right)=(-1)^{d-k}(k-j) ! S(k+1, k+1-j)$ for every integer $j$ with $0 \leqslant j \leqslant k$. Hence we get $e_{j}^{k, d}\left(t^{k}\right)=(-1)^{d-k}(d-$ $j) ! S(k+1, d+1-j)$ for every integer $j$ with $d-k \leqslant j \leqslant d$. On the other hand, we have $e_{j}^{k, d}\left(t^{k}\right)=0=(-1)^{d-k}(d-j) ! S(k+1, d+1-j)$ for every integer $j$ with $0 \leqslant j \leqslant d-k-1$. So we get the assertion. 
Corollary 2. Let $d$ be a nonnegative integer and $S(a, b)$ the Stirling number of the second kind. Then for every integer $j$ with $0 \leqslant j \leqslant d$ we have

$$
e_{j}\left(t^{d}\right)=(d-j) ! S(d+1, d+1-j) .
$$

Theorem 3. Let $d$ be a nonnegative integer and $h(t) \in \mathbb{C}[t]$ be a polynomial $h(t)=\sum_{k=0}^{d} c_{k} t^{k}$ in $t$ such that $h(n) \in \mathbb{Z}$ for every integer $n$, where $c_{d} \neq 0$. Then for every integer $i$ with $0 \leqslant i \leqslant d$ the following equality holds.

$$
\chi_{i}^{H}(h)=\sum_{l=0}^{d}(-1)^{l} c_{d-l}\left\{\sum_{j=0}^{i}(-1)^{j}(d-j) ! S(d+1-l, d+1-j)\right\} .
$$

Proof. First we note that $t^{k}=\sum_{j=0}^{d}(-1)^{j} e_{j}^{k, d}\left(t^{k}\right)\left(\begin{array}{c}t+d-j \\ d-j\end{array}\right)$. Then

$$
\begin{aligned}
h(t) & =\sum_{k=0}^{d} c_{k} t^{k}=\sum_{k=0}^{d} c_{k}\left\{\sum_{j=0}^{d}(-1)^{j} e_{j}^{k, d}\left(t^{k}\right)\left(\begin{array}{c}
t+d-j \\
d-j
\end{array}\right)\right\} \\
& =\sum_{j=0}^{d}(-1)^{j}\left\{\sum_{k=0}^{d} c_{k} e_{j}^{k, d}\left(t^{k}\right)\right\}\left(\begin{array}{c}
t+d-j \\
d-j
\end{array}\right) \\
& =\sum_{j=0}^{d}(-1)^{j}\left\{\sum_{l=0}^{d} c_{d-l} e_{j}^{d-l, d}\left(t^{d-l}\right)\right\}\left(\begin{array}{c}
t+d-j \\
d-j
\end{array}\right) .
\end{aligned}
$$

So we get $e_{j}(h(t))=\sum_{l=0}^{d} c_{d-l} e_{j}^{d-l, d}\left(t^{d-l}\right)$ (see Notation 4). Hence

$$
\chi_{i}^{H}(h)=\sum_{j=0}^{i}(-1)^{j}\left\{\sum_{l=0}^{d} c_{d-l} e_{j}^{d-l, d}\left(t^{d-l}\right)\right\} .
$$

By using Theorem 2 we have

$$
\begin{aligned}
\chi_{i}^{H}(h) & =\sum_{j=0}^{i}(-1)^{j}\left\{\sum_{l=0}^{d} c_{d-l}(-1)^{l}(d-j) ! S(d-l+1, d+1-j)\right\} \\
& =\sum_{l=0}^{d}(-1)^{l} c_{d-l}\left\{\sum_{j=0}^{i}(-1)^{j}(d-j) ! S(d+1-l, d+1-j)\right\} .
\end{aligned}
$$

Therefore we get the assertion. 
By Theorem 3 we see that if $h(t)=t^{d}$, then

$$
\chi_{i}^{H}(h)=\sum_{j=0}^{i}(-1)^{j}(d-j) ! S(d+1, d+1-j) .
$$

In this case, we can also prove the following theorem.

Theorem 4. Let d be a nonnegative integer and $S(a, b)$ the Stirling number of the second kind, where $a$ and $b$ are nonnegative integers. Let $h(t)=t^{d}$. Then for every integer $i$ with $0 \leqslant i \leqslant d$ we have

$$
\chi_{i}^{H}(h)=(-1)^{i}(d-i) ! S(d, d-i) .
$$

Proof. We prove this by induction on $i$.

(i) If $i=0$, then $\chi_{0}^{H}(h)=e_{0}\left(t^{d}\right)=d ! S(d+1, d+1)=d ! S(d, d)$ and this shows that it is true for the case of $i=0$.

(ii) Assume that it is true for the case of $i=k-1$. So we have

$$
\sum_{j=0}^{k-1}(-1)^{j} e_{j}\left(t^{d}\right)=\chi_{k-1}^{H}(h)=(-1)^{k-1}(d-k+1) ! S(d, d-k+1) .
$$

Next we consider the case of $i=k$. Then by Corollary 2, [6, (1.93)] and (3)

$$
\begin{aligned}
\chi_{k}^{H}(h)= & \sum_{j=0}^{k}(-1)^{j} e_{j}\left(t^{d}\right)=(-1)^{k} e_{k}\left(t^{d}\right)+\sum_{j=0}^{k-1}(-1)^{j} e_{j}\left(t^{d}\right) \\
= & (-1)^{k}(d-k) ! S(d+1, d+1-k) \\
& \quad+(-1)^{k-1}(d-k+1) ! S(d, d+1-k) \\
= & (-1)^{k-1}(d-k) !\{-S(d+1, d+1-k) \\
& \quad+(d-k+1) S(d, d+1-k)\} \\
= & (-1)^{k-1}(d-k) !\{-(d+1-k) S(d, d+1-k) \\
& \quad-S(d, d-k)+(d-k+1) S(d, d+1-k)\} \\
= & (-1)^{k}(d-k) ! S(d, d-k) .
\end{aligned}
$$

So we get the assertion.

The following corollary of Theorem 4 has been proved in [3] by a different method. 
Corollary 3 (Kaki). Let $P$ be a poset such that $P$ has no order. Then for every integer $i$ with $0 \leqslant i \leqslant d(P)$ we have

$$
g_{i}(P)=(d(P)-i) ! S(d(P), d(P)-i) .
$$

Proof. Here we note that $h_{P}(t)=t^{d(P)}$ in this case. By Theorem 4 we have $\chi_{i}^{H}(P)=\chi_{i}^{H}\left(h_{P}\right)=(-1)^{i}(d(P)-i) ! S(d(P), d(P)-i)$. On the other hand,

$$
\nabla f_{2}(0)=\sum_{y \leqslant 0} f_{2}(y)=\sum_{y \leqslant 0} h_{P}(0) \rho(y)=h_{P}(0) \rho(0)=h_{P}(0)=0
$$

and

$$
\begin{aligned}
\nabla f_{2}(-i) & =\sum_{y \leqslant-i} f_{2}(y)=\sum_{y \leqslant-i} h_{P}(0) \rho(y) \\
& = \begin{cases}h_{P}(0) \rho(0), & \text { if } i=0, \\
0, & \text { if } i \geqslant 1\end{cases} \\
& =0 .
\end{aligned}
$$

Therefore by Definition 4 we get the assertion.

Theorem 5. Let $P$ be a finite poset. Then the following are equivalent each other.

(i) $P$ has no order.

(ii) $g_{d(P)-1}(P) \neq 0$.

(iii) $g_{d(P)-1}(P)=1$.

Proof. By Theorem 1 we see that $g_{d(P)-1}(P) \neq 0$ if and only if $l(P)<$ $d(P)-(d(P)-1)=1$. Hence $g_{d(P)-1}(P) \neq 0$ if and only if $l(P)=0$. So we get the equivalence (i) and (ii) because $l(P)=0$ means that $P$ has no order.

On the other hand, by Corollary 3 we see that

$$
g_{d(P)-1}(P)=S(d(P), 1)=1
$$

if $P$ has no order. Therefore (i) implies (iii). Since (iii) implies (ii), we get the assertion.

Theorem 6. Let $P$ be a finite poset. Then

$$
g_{i}(P) \leqslant(d(P)-i) ! S(d(P), d(P)-i)
$$

holds for every integer $i$ with $0 \leqslant i \leqslant d(P)-1$. Moreover this equality holds for some $i$ with $0 \leqslant i \leqslant d(P)-1$ if and only if $P$ has no order. 
Proof. Here we note the following

Claim 1. Let $P$ be a finite poset. Then $P$ has an order if and only if $\sigma \notin \mathcal{L}(P)$, where

$$
\sigma=\left(\begin{array}{ccccc}
1 & 2 & \cdots & d(P)-1 & d(P) \\
d(P) & d(P)-1 & \cdots & 2 & 1
\end{array}\right)
$$

Proof. First we note that $S_{d(P)}=\mathcal{L}(P)$ if $P$ has no order. Hence $\sigma \in \mathcal{L}(P)$ if $P$ has no order. So it suffices to show that $\sigma \notin \mathcal{L}(P)$ if $P$ has an order. Assume that $P$ has an order. Then there exist elements $x_{i}, x_{j} \in P$ such that $x_{i}<x_{j}$. For any $\delta \in \mathcal{L}(P)$, there exist $\mu, \pi \in A(P)$ such that $\delta=\pi \circ \mu^{-1}$. On the other hand, we have $\mu\left(x_{i}\right)=a_{1}<b_{1}=\mu\left(x_{j}\right)$ and $\pi\left(x_{i}\right)=a_{2}<b_{2}=\pi\left(x_{j}\right)$. Then we have $\delta\left(a_{1}\right)=a_{2}<b_{2}=\delta\left(b_{1}\right)$. Since $\sigma$ does not satisfiy this property, we see that $\sigma \notin \mathcal{L}(P)$ and we get the assertion of this claim.

Using this claim, we can prove the following.

Claim 2. Let $Q$ and $R$ be finite posets with $d(Q)=d(R)$. Assume that $Q$ has no order and $R$ has an order. Then $g_{i}(Q)>g_{i}(R)$ for every integer $i$ with $0 \leqslant i \leqslant d(Q)-1=d(R)-1$.

Proof. We use Notation 5. First of all, we note that $\mathcal{L}(Q)=S_{d(Q)}$. We see from Proposition 1 that $d\left(\phi_{f_{Q}}\right)=d(Q)$. So by Remark 3 (1) we have

$$
g_{i}(Q)=\sum_{k=i+1}^{d(Q)}\left(\begin{array}{c}
k-1 \\
i
\end{array}\right) a_{k}(Q)
$$

for every integer $i$ with $0 \leqslant i \leqslant d(Q)-1$. We note that $a_{d(Q)}(Q)>0$ by Proposition 1 and Claim 1.

On the other hand, $\mathcal{L}(R) \subset S_{d(R)}=S_{d(Q)}=\mathcal{L}(Q)$ holds by assumption. We see from Proposition 1 and Claim 1 that $d\left(\phi_{f_{R}}\right)<d(R)=d(Q)$. We note that by Proposition 1 we obtain $a_{k}(Q) \geqslant a_{k}(R)$ for every integer $k$ with $0 \leqslant k \leqslant d(Q)=d(R)$. Hence we see from Proposition 1 that

$$
\begin{aligned}
g_{i}(Q)-g_{i}(R) & =\sum_{k=i+1}^{d(Q)}\left(\begin{array}{c}
k-1 \\
i
\end{array}\right) a_{k}(Q)-\sum_{k=i+1}^{d\left(\phi_{f_{R}}\right)}\left(\begin{array}{c}
k-1 \\
i
\end{array}\right) a_{k}(R) \\
& \geqslant a_{d(Q)}(Q)>0 .
\end{aligned}
$$

Therefore we get the assertion of Claim 2.

By Claim 2 and Corollary 3, we get the assertion of Theorem 6. 


\section{References}

[1] Y. Fukuma, On invariants of polynomial functions, Japan. J. Math 31 (2005), 345-378.

[2] Y. Fukuma, Topics on invariants of polarized varieties, Sugaku Expositions 25 (2012), 19-45.

[3] Y. Kaki, Master's thesis, Kochi University, in Japanese (2007).

[4] A. Ooishi, Genera and arithmetic genera of commutative rings, Hiroshima Math. J. 17 (1987), 47-66.

[5] A. Ooishi, $\Delta$-genera and sectional genera of commutative rings, Hiroshima Math. J. 17 (1987), 361-372.

[6] R. P. Stanley, Enumerative combinatorics, Volume 1, Second edition. Cambridge Studies in Advanced Mathematics, 49. Cambridge University Press, Cambridge, 2012 .

\section{CONTACT INFORMATION}

Yoshiaki Fukuma Department of Mathematics and Physics, Faculty of Science and Technology, Kochi University, Akebono-cho, Kochi 780-8520, Japan E-Mail(s): fukuma@kochi-u.ac.jp

Received by the editors: 15.01.2019

and in final form 02.06.2020. 